\title{
EDITORIAL
}

\section{INCENTIVOS EN ATENCIÓN PRIMARIA: DE LA CONTENCIÓN DEL GASTO A LA SALUD DE LA POBLACIÓN}

\author{
Juan Gérvas, Vicente Ortún, Luis Palomo, Miguel Ángel Ripoll, y Seminario de Innovación en \\ Atención Primaria 2007
}

\section{INTRODUCCIÓN}

A finales del siglo XIX empezó a separarse la gestión de la propiedad de la misma, y a principios del siglo XX empezó a ser relevante conseguir que el gestor y el trabajador hicieran lo que se esperaba de ellos (coincidir en los objetivos con el propietario). Es decir, comenzó la psicología industrial y el interés científico por la motivación de las personas. Después de casi cien años de estudios puede decirse algo obvio y simple: los gestores y trabajadores responden globalmente a los incentivos por tener impulsos, razones y valores que les llevan a implicarse (o inhibirse) en las tareas que se les asignan. Los incentivos se engloban en el amplio campo de la motivación, las complejas razones que rigen la conducta humana, cuestión clave en la gestión de todas las empresas ${ }^{1-5}$.

En atención primaria lo clave es buscar la consecución integral, pues si se miden "cosas" (que es lo fácil), se harán "cosas" ( $\sin$ poder asegurar que se haga lo que se debe hacer). Se trata de medir la calidad no

Correspondencia:

Juan Gérvas

Equipo CESCA

Travesía de la Playa, 3

Buitrago del Lozoya. 28730. Madrid

Teléfono: 918680182

Correo electrónico: jgervasc@meditex.es la cantidad, pues lo que importa es la salud $^{6}$, y en primaria la atención global del paciente y de la población. Por ejemplo, si sólo se mide el desempeño en la atención a las personas diabéticas por el nivel de hemoglobina glicosilada se lograrán buenas marcas en tal indicador, pero la cuestión no es tan sencilla, pues el objetivo final es conseguir menos amputaciones, cegueras, comas hipoglucémicos, infartos de miocardio e insuficiencias renales, atenderlas adecuadamente en sus otros problemas de salud, y lograr una distribución del tiempo de atención y de los recursos empleados en diabéticos que maximice beneficios y disminuya riesgos, en ellos y en el conjunto de los pacientes atendidos y de la población "cubierta". Este ejemplo demuestra que, aunque sea posible y necesario, no es tarea fácil incentivar y motivar a los médicos de atención primaria. Los problemas de medición del rendimiento de los médicos son tales que cabe preguntarse por las alternativas a los incentivos (cambios en el marco institucional, modificaciones de la organización, carrera profesional, mejoras en la selección del personal, cambios en la forma de trabajo, etcétera) $)^{3,7}$.

Los incentivos son monetarios, pero también de otros muchos estilos, desde promoción interna a baterías personalizadas de mejoras en las condiciones de trabajo, por ejemplo. Los incentivos pueden ayudar a racionalizar el gasto y a motivar al médico, 
y su eficacia estará en función del diseño. Aquello que se incentive movilizará al profesional y siempre tendrá, en mayor o menor grado, efectos secundarios que deben considerarse. Lamentablemente, sabemos poco sobre la efectividad de los incentivos en atención primaria, porque no es fácil dar respuesta a problemas complejos y, entre otras cosas prácticas, por la falta de publicaciones sobre su uso en las Comunidades Autónomas españolas ${ }^{4,8}$. Así pues, respecto a incentivos en primaria, conviene la reflexión, la prudencia y la publicación.

En este texto analizamos algunas cuestiones en torno a los incentivos en atención primaria desde el punto de vista del médico clínico. Partimos de lo más frecuente y habitual, la contención del gasto, para llegar al objetivo último, la mejora de la salud de la población con énfasis en el uso diagnóstico y terapéutico de la silla (de los procedimientos que los médicos de primaria pueden realizar en sus propias consultas, en el domicilio del paciente y/o en la comunidad) Hemos escogido el término "silla" por la frase atribuida a Marañón del "valor diagnóstico y terapéutico de la silla", referido a la escucha, a la historia clínica y a la exploración física.

\section{CONTENCIÓN DEL GASTO}

En la práctica, muchas veces los incentivos cumplen objetivos de contención del gasto y se ha demostrado que en ocasiones tal política es acertada; es decir menos (gasto) es mejor (salud). Por ejemplo, respecto a la hipertensión el gasto se puede reducir a la sexta parte cuando se trabaja con calidad ${ }^{9}$. Desde luego, no se pretende el ahorro sin más, sino el mejor uso de los recursos disponibles, pues además el presupuesto aprobado en el sector público es para gastarlo íntegramente. Lo importante es el cómo y en qué, más que el cuánto (gastamos $)^{6}$. Los gestores tienen un presupuesto para alcanzar un fin y los médicos clínicos un compromiso para lograr el mejor uso del gasto que su actividad genera.

El gasto sanitario español puede crecer con prudencia. Lo importante es lograr que la intensidad de la atención (número de actividades por episodio) se ajuste a la necesidad ${ }^{10}$. Por ejemplo, el dolor abdominal "funcional" típico del niño y del adolescente suele ser un proceso autolimitado que se resuelve por sí mismo en más de la mitad de los $\operatorname{casos}^{11}$ y puede manejarse generalmente en la consulta del médico general ${ }^{11,12}$ ("la silla"), pero en la persecución del diagnóstico orgánico imposible se pueden cruzar innecesariamente los límites de lo razonable (derivación al gastroenterólogo e incluso ingreso hospitalario, y análisis de sangre con marcadores y de orina con cultivo, ecografía abdominal y endoscopia gastrointestinal alta, vía oral, y baja, anal, con biopsia de la mucosa del íleo, por ejemplo $)^{13}$.

La clave no es la contención del gasto sino la adecuación y calidad de las prestaciones. Es decir, lograr que se haga lo que se puede hacer y no se suele hacer en las condiciones diarias de la práctica clínica. En otras palabras, la clave es ir tendiendo puentes que ayuden a salvar el abismo que separa la eficacia de la efectividad ${ }^{6,14}$. Efectividad clínica y eficiencia social (trabajar bien y priorizar lo que se hace). Lo segundo, priorizar, es más difícil hacerlo con fundamento, en muchos casos por falta de conocimiento científico suficiente respecto a la comparación entre alternativas, como se demuestra bien en el caso de la prevención clínica ${ }^{15,16}$.

Se trata de lograr efectividad y seguridad y ello implica un ideal: el uso adecuado en el $100 \%$ de los pacientes que precisan una intervención, y el no uso de intervenciones no necesarias en el $100 \%$ de los pacientes que no las precisan ${ }^{17}$. Por ejemplo, la solicitud apropiada de pruebas (análisis y otras), la necesaria coordinación de la aten- 
ción en los pacientes con múltiples y simultáneos problemas de salud, el empleo adecuado de los antibióticos, o la mejor organización de las visitas a domicilio a los pacientes crónicos invalidados. Estos logros son ética y profesionalmente exigibles, pues llevan a la práctica de una buena medicina, a un trabajo de excelencia, y los incentivos para promoverlos siempre encontrarán eco en los médicos clínicos ${ }^{18}$.

Los incentivos explícitos de simple contención del gasto (bonos por cumplimiento presupuestario en farmacia, por ejemplo) pueden llegar a destruir incentivos implícitos (autoestima por el uso racional de los medicamentos, por ejemplo), y a disminuir la efectividad y la seguridad. De hecho, la hostilidad frente a incentivos "economicistas", en la jerga y percepción de los médicos, puede llevar a la disminución de la cooperación en el logro de objetivos de salud e incluso a la pérdida del gusto por el trabajo bien hecho (lo que aboca al "quemarse" en el día a día clínico).

Además, la contención del gasto no conlleva necesariamente equidad. Por ejemplo, en el control del gasto farmacéutico es importante recordar que los españoles de clase baja tienen triple mortalidad por causa infecciosa ${ }^{19}$, lo que obliga a la mejora de la accesibilidad y a un uso diferencial de los antibióticos y de otras intervenciones (como la radiografía de tórax) según clase social. De nuevo no se trata de la simple contención del gasto, sino del mejor uso de los recursos, del aumento de la efectividad, eficiencia, seguridad y equidad.

En el uso de su propio tiempo y de los otros recursos disponibles, el médico suele trabajar casi sin darse cuenta del doble papel que ejerce ante el paciente y la sociedad (el eterno debate entre la irracionalidad técnica y la irracionalidad romántica) ${ }^{20,21}$. En su trabajo diario se confunde la gestión de la decisión (recogida de información y formulación de alternativas) con el control de la decisión (elección de la alternativa y monitorización de los efectos). Lo habitual en los trabajos por cuenta ajena es la separación entre ambas funciones (y en el sector público la fiscalización previa del gasto), que apenas se da en las organizaciones sanitarias.

\section{INGENIERÍA DOCUMENTAL}

La motivación y los incentivos buscan la armonización entre los objetivos de la organización y los de los trabajadores ${ }^{1-5}$. En el sistema sanitario español los desencuentros y discrepancias dependen tanto de la indefinición de la organización (y/o de sus "agendas ocultas") como de los intereses peculiares de médicos funcionarios. Cuando no hay motivación y los incentivos se aceptan como complemento salarial, cuando comprometen a poco, caben conductas de cumplimiento formal. Así, se trata de cumplir con los requisitos que aseguran la consecución de los incentivos, bien dedicando tiempo excesivo a las tareas que "dejan huella", bien incluso dedicando tiempo exclusivo a "dejar huella" (donde "huella" es el registro de la actividad incentivada) ${ }^{22}$. Con ello se entra en un auténtico juego de comportamientos oportunistas de algunos profesionales "aprovechados" ante alguna organización "hipócrita" (que tiene objetivos teóricos y prácticos incongruentes) ${ }^{2}$, y el registro se transforma de actividad periférica en central. El médico y la organización pierden mucho en este camino y los pacientes y la sociedad pierden más.

La "ingeniería documental" se evitaría en mucho si los incentivos se basasen en indicadores extraídos de registros consustánciales a la práctica clínica de calidad. Es decir, si los indicadores no precisasen de registros específicos que detraigan tiempo clínico o que den oportunidad de acicalarlos (o inventarlos, incluso). Además, siempre que sea posible los indicadores básicos podrían complementarse con 
indicadores externos, más centrados en el resultado $^{23}$. Por ejemplo, con el uso prudente de información de la que ya se dispone en España tipo "hospitalizaciones evitables por primaria", "mapas de variabilidad de la práctica clínica" y otras no probadas, como por ejemplo el número de pacientes que mueren en las ambulancias (posible indicador de coordinación en la atención a los pacientes terminales), aunque con la idea clara de que el bajo número de casos no aconseja el uso de estos indicadores en médicos individuales ${ }^{24}$.

Con ello se evitaría el aspecto más grosero de la ingeniería documental, pero no el efecto de "lupa" de los incentivos, que lleva a concentrar el esfuerzo clínico en aquello que se sabe se medirá. Este efecto, aunque cierto y demostrado, no siempre se ha asociado al deterioro de la calidad de lo "no medido" $" 25-27$.

El efecto lupa se evita con una política global que fomente el buen trabajo, premie y sostenga a los que ya lo hacen bien, estimule a los que puedan mejorar e introduzca los correctivos necesarios para que los médicos se sientan positivamente discriminados. Por el lado de los profesionales se precisa de un liderazgo, de valores y de compromiso, que se echa de menos en el contexto español donde en ocasiones el ambiente profesional dista mucho del ideal $^{28}$.

\section{LA SALUD DEL PACIENTE (Y DE LA POBLACIÓN)}

Los médicos, especialmente los de cabecera, cuentan con el aprecio de la población, pues contribuyen substancialmente a incrementar la salud de los pacientes, lo que es cierto en algunos casos (desde el simple tratamiento de un dolor de espalda al consejo contra el tabaquismo) pero no siempre. De hecho, las intervenciones médicas innecesarias se han convertido en un verdadero problema de salud pública ${ }^{29}$. Por ello, lo incentivos tienen tanto sentido cuando promueven la adopción de nuevas intervenciones eficaces como cuando evitan la introducción o persistencia de actividades innecesarias.

Se trata de tener como objetivo la salud del paciente y de la población, no la carga de trabajo ${ }^{16,23}$. Esta es muchas veces excesiva por el brillo de la tecnología "dura", instrumental ${ }^{30}$, en detrimento de la tecnología "blanda", cognitiva.

La simple búsqueda de calidad (de efectividad) sin cantidad tasada puede ser perjudicial para la seguridad y la equidad ${ }^{31,32}$. Si lo que preocupa es la salud de los pacientes, y en general de la población, el objetivo de los incentivos, y de una "cartera de servicios" científica es conseguir disminuir el número medio de intervenciones diagnósticas y terapéuticas por problema de salud por episodio atendido. Esta regla general es de dificilísima aplicación práctica, pues ha de adaptarse al contexto del paciente y de la población, pero si se consigue se logra al tiempo la disminución de la carga de trabajo y la mejora de la salud, y se estarían liberando recursos que permitirían mejorar la calidad de los servicios (que se ofrecen a los ciudadanos, financiadores y propietarios de los mismos).

En ese sentido los incentivos que promuevan la disminución y adecuación del número de actividades diagnósticas y terapéuticas a las necesidades del paciente concreto contarán siempre con la aprobación de los médicos, pues forman parte de su viejo acervo básico, el primum non nocere.

Se consigue además impacto en la población cuando las actividades se llevan a cabo en quienes más las necesitan, mediante la mejora de la accesibilidad, que repercute en la equidad ${ }^{33}$. Así, por ejemplo, a través de la disminución de recursos para los pacientes "okupas" y del aumento de los mismos para 
los grupos marginales (entre estos se encuentran desde los que tienen simples problemas horarios, como los trabajadores, a los que pertenecen a grupos sociales excluidos, como drogadictos y prostitutas, pues son muchos los que tienen problemas crecientes de accesibilidad a su médico, con "colas" de hasta quince días para lograr cita, y con uso vicariante de las urgencias).

Los objetivos, pues, son cuatro: efectividad, eficiencia, seguridad y equidad. En ellos creen los médicos y con su consecución es fácil lograr la cooperación entre clínicos y gestores, y la armonización de los objetivos de la organización con los objetivos de los profesionales. Los objetivos comunes son: "máxima calidad, mínima cantidad, tecnología apropiada y adecuada a la necesidad, y tan cerca del paciente como sea posible" 29,31 .

Lo que importa es el impacto en la salud de los pacientes y de la población ${ }^{6,16,23}$. De ello sabemos poco, pero tenemos certeza, por ejemplo, de que es mucho mayor el "rendimiento en salud" de la introducción del IECA en el paciente con insuficiencia cardiaca que el seguimiento durante años del hipertenso; y de que es mucho mayor el impacto en salud de la población del consejo por el médico general contra el tabaco que la prevención primaria y secundaria de la isquemia de miocardio con hipolipemiantes ${ }^{16}$. Este es el tipo de información que puede soportar incentivos centrados en la salud.

\section{LA SILLA}

Denominamos "silla" al conjunto de procedimientos que los médicos de primaria pueden realizar en sus propias consultas, en el domicilio del paciente y/o en la comunidad, con énfasis en lo básico (entrevista clínica y exploración física, escucha terapéutica, empatía, apoyo, consejo, espera expectante y demás).
Toda la estrategia de incentivos en torno a la disminución y adecuación de las actividades diagnósticas y terapéuticas debería descansar en la mejora de la capacidad de resolución del médico de cabecera, basada en la polivalencia de su trabajo, su tecnología "blanda", cognitiva, y en lo que es propio de la atención primaria (longitudinalidad, integralidad, accesibilidad y continuidad) ${ }^{2,4,34-39}$. Es decir, en la práctica depende básicamente de emplear los incentivos para devolver el brillo tecnológico a la "silla".

Además de la implantación de incentivos ad hoc se puede devolver el brillo tecnológico a la "silla" con intervenciones varias. A destacar:

Investigación. Sobre todo, en torno a lo que sucede a diario en la consulta, al control de la incertidumbre y al método de trabajo del médico de familia (que puede llegar a resolver la mitad de los problemas atendidos sin un diagnóstico final). Sabemos poco sobre estas cuestiones, centrales en un trabajo basado en la "silla".

Formación. Durante el pregrado, postgrado y formación continuada, cuanto más centrada esté la docencia en la atención primaria mejor; con el uso docente de los especialistas como consultores (por su énfasis en el diagnóstico, por su falta de control de la incertidumbre, por la anómala prevalencia de enfermedad en su entorno, y por la fascinación tecnológica de su práctica clínica).

Profesionalismo. El desarrollo de valores $\mathrm{y}$ actitudes que lideren las sociedades profesionales o grupos de médicos decididos a instaurar la ética de la excelencia. La búsqueda y el fomento de reglas claras del juego con la organización y con la sociedad, de forma que sea norma la transparencia en el ejercicio profesional orgulloso del buen hacer clínico. 
Apoyo gerencial. Gran parte de la reputación clínica depende de la existencia de una gerencia profesional, relativamente independiente de los avatares políticos, $\mathrm{y}$ bien entrenada para facilitar el trabajo diario con los medios disponibles.

Definición de objetivos en salud de la población, y atribución de posibilidades según niveles, con incentivos para lograrlo. La morbilidad y la mortalidad sanitariamente evitables se pueden definir, y también se pueden calcular las posibilidades y la contribución del trabajo de cada nivel y de cada actividad para reducirlas. De esta forma se vuelven operativos y se pueden incentivar, los objetivos de "máxima calidad, mínima cantidad, tecnología apropiada y adecuada a la necesidad, y tan cerca del paciente como sea posible".

Reorganización de la práctica clínica. Con ensayos bien evaluados de otras formas de contrato, de prestación de servicios y de organización que den más autonomía, autoridad y responsabilidad al médico de cabecera.

Introducción de pequeñas dosis de tecnología "dura" (instrumental) conveniente en la consulta del médico de cabecera. Aunque el objetivo final es quitar brillo a la tecnología "dura" diagnóstica y terapéutica, dosis mínimas de la misma puede contribuir a dar atractivo a la "silla". Así, por ejemplo, el fácil y fluido contacto telefónico con los especialistas, el acceso a pruebas diagnósticas, una historia clínica electrónica avanzada (con ayuda a la decisión, por ejemplo), material adecuado para la cirugía menor y la ginecología, la dotación de ecógrafo y de cámara digital (para el estudio del fondo de ojos en diabéticos), la mejora de la accesibilidad con el propio médico de cabecera (consulta telefónica y electrónica) y otros avances que aumenten la capacidad de resolución del médico de primaria. Se precisa, evidentemente, de incentivos y correctivos, de un control ex post permanente del número total de actividades diagnósticas y terapéuticas por episodio de atención, pues lo que se busca es la adecuación a la necesidad.

Desde luego, habría que lograr eco en los medios de comunicación, tan centrados en lo tecnológico y en los "errores médicos", y reivindicar con ejemplos el eficiente empleo diagnóstico y terapéutico de la "silla", así como su impacto en la protección del paciente ante el uso abusivo e innecesario de muchos procedimientos y tratamientos.

Adicionalmente, ayudaría a sostener la "silla" lograr alejar el fantasma de las "reclamaciones judiciales", pues el miedo al abandono ante las mismas lleva a una medicina defensiva plena de actividades diagnósticas y terapéuticas excesivas y/o innecesarias. Un sistema de arbitraje, previo al judicial, podría poner las cosas en su sitio, y ayudar a restablecer la lógica, al tiempo que permitiría reparar el daño y aprender de los errores, mientras el médico de familia se sentiría respaldado y apoyado en su forma de trabajo.

Si el objetivo del sistema sanitario es producir salud (disminuir la morbilidad y mortalidad innecesariamente prematura y sanitariamente evitable, desde el parto digno a la muerte digna, con independencia del lugar en el que acontezca) la "silla" debe recuperar atractivo para el médico clínico. Parte de ese atractivo depende de la existencia de incentivos y correctivos adecuados. Mucho depende de otras cuestiones, como el liderazgo de un profesionalismo que hoy se echa en falta en España.

\section{AGRADECIMIENTOS}

Este texto se elaboró a partir del debate en el segundo taller presencial de los 
Seminarios de Innovación en Atención Primaria, que en 2007 se dedican a la organización de los servicios en atención primaria. Se celebró en Madrid, el 22 de junio de 2007, y fue ponente Vicente Ortún, contrapuntos Luis Palomo y Miguel Ángel Ripoll, y coordinador Juan Gérvas. Los Seminarios de Innovación en Atención Primaria están organizados por la Fundación Ciencias de la Salud y la Fundación para la Formación de la Organización Médica Colegial, y cuentan con el patrocinio de GSK. A los participantes en los debates previos y posteriores del segundo Seminario de Innovación 2007, que también generaron ideas durante el debate presencial. Fueron participantes en dicho seminario: Abarca B, Amengual M, Ayala V, Bonis J, Casajuana J, Coll Benejam JM, Domínguez Velázquez J, Ezquerra M, García Marco CM, García Olmos LM, Gómez Moreno N, Martínez T, Minué S, Morera R, Otero A, de Pablo R, Palacín JC, Pastor-Sánchez R, Pérez Artigues JA, Pérez Fernández M, Rico A, Redondo J, Rivas Costa G, Rodríguez Ledo P, Rodríguez Sendín JJ, Ruiz Téllez A, Simó J, Timoner J, Vázquez Díaz JR, Saffron JP, Solla JM, Vela Cordón P, Vicente Molinero A, Violan C.

\section{BIBLIOGRAFÍA}

1. Milgrom P, Roberts J. Economía, organización y gestión de la empresa. Barcelona: Ariel; 1993.

2. Simó J, Chinchilla N. Motivación y médicos de familia (II). Aten Primaria. 2001;28:668-73.

3. Roberts J. The modern firm. Organizational design for performance and growth. Oxford: OUP; 2004.

4. Martín JJ. Motivación, incentivos y retribuciones de los médicos de atención primaria en el Sistema Nacional de Salud. Rev Adm Sanit. 2005;3:111-30.

5. Casadesús R, Spulber D. Agency revisited. Madrid: Fundación BBVA; 2007.

6. Ortún V. Mejor (servicio) es más (calidad). Rev Esp Salud Pública. 2006;60:267-71.
7. Simó J, Campos JC, Sanfélix J. Carrera profesional y médicos de familia: reflexiones propuesta. Aten Primaria. 2002;29:172-7.

8. Díaz Ojeda E, Navarro P, Prados S. Sistema Nacional de Salud: la retribución variable. Rev Adm Sanit. 2005;3:205-35.

9. Bonet A, Gosalbes V, Fito M, Navarro J. Prescripción racional y reducción de costes en el tratamiento de la hipertensión arterial: un ejercicio de simulación. Gac Sanit. 2001;15:327-35.

10. Puig-Junoy J. ¿Es la financiación sanitaria suficiente y adecuada? Gac Sanit. 2006;20 (Supl 1): 96-102.

11. Muris JWM, Starman R, Fijten G, Knottnerus JA. One-year prognosis of abdominal complaints in general practice: a prospective study in patients in whom non organic cause is found. Br J Gen Pract. 1996;46:715-9.

12. Edwards MW, Forman WM, Walton J. Audit of abdominal pain in general practice. J R Coll Gen Pract. 1985;35:235-8.

13. Lindley KJ, Glasser D, Mills PJ. Consumerism in healthcare can be detrimental to child health: lessons form children with functional abdominal pain. Arch Dis Child. 2005;90:335-7.

14. Institute of Medicine. Crossing the quality chasm: a new health system for the 21 th century. Washington: National Academic Press; 2001.

15. Maciosek MV, Coffield AB, Edward NM, Flottemesh TJ. Goodman MJ, Solberg CI. Priorities among effective clinical preventive services. Results of a systematic review and analysis. Am J Prev Med. 2006;31:52-61.

16. Fleetcroft R, Cookson R. Do the incentive payments in the new NHS contract for primary care reflect likely population health gains? J Health Serv Res Policy. 2006;11:27-31.

17. Reilly BM, Evans AT. Translating clinical research into clinical practice. Impact of using prediction rules to make decisions. Ann Intern Med. 2006;144:201-9.

18. Tevés J, Salmerón JM, Blanco JA, Calvet C, Morlans M. La direcció per objectives incentivats. Un dilema deontológico? Annals Med. 2007;90:35-7.

19. Regidor E, Mateo S, Calle ME, Domínguez V. Educational level and mortality from infectious diseases. J Epidemiol Community Health. 2002;56:682-3. 
20. Hampton JR. The end of clinical freedom. BMJ. 1983;287:1237-8.

21. Abrams FR. The doctor with two heads. N Engl J Med. 1993;328:975-6.

22. Ripoll MA. ¿Es eficiente la Cartera de Servicios como herramienta de mejora de calidad? Med Gen. 2000;27:794-6.

23. Soto J. Medicina basada en resultados de salud; la evolución lógica de la medicina basada en pruebas. Med Clín (Barc). 2007;128:254-5.

24. Gérvas J, Caminal Homar J, Seminario Innovación 2006. Las hospitalizaciones por Ambulatory Care Sensitive Conditions (ACSC) desde el punto de vista del médico de atención primaria. Rev Esp Salud Pública. 2007;81:7-13.

25. McGlynn EA. Entended and unitended consequences. What should we really worry about? Med Care. 2007;45:3-5.

26. Ganz DA, Wenger NS, Roth CP, Kamberg CJ, Chang JT et al. The effect of a quality improvement initiative on the quality of other aspects of health care. The law of unintended consequences? Med Care. 2007;45:8-18.

27. Steel N, Maisey S, Clark A, Fleetcroft R, Howie A. Quality of primary care and targeted incentive payments: an observational study. Br J Gen Pract. 2007;57:449-54.

28. Pardell H, Gual A, Oriol A. ¿Qué significa ser médico hoy? Med Clín (Barc). 2007;129:17-22.

29. Gérvas J. La clave para mejorar la clínica: más calidad con el mínimo de cantidad. Gac Sanit Bilbao. 2006;103:46-7.
30. Peiró S, Bernal E. ¿A qué incentivos responde la utilización hospitalaria en el Sistema Nacional de Salud? Gac Sanit. 2006;20 (Supl 1):110-6.

31. Gérvas J. Moderación en la actividad médica preventiva y curativa. Cuatro ejemplos de necesidad de prevención cuaternaria. Gac Sanit. 2006;20 (Supl 1):127-34.

32. David P. Health care as a risk factor. CMAJ. 2004;170:1688-9.

33. Tudor JT. The inverse care law. Lancet. 1971; I:405-12.

34. Gérvas J, Pané O, Sicras A, Seminario Innovación Atención Primaria 2006. Capacidad de respuesta de la atención primaria y buena reputación profesional, algo más que buen trabajo clínico. Med Clín (Barc). 2007;128:540-4.

35. Gérvas J, Ortún V. Propuesta de incentivos para una medicina general por cuenta ajena. Gac Sanit. 1996;10:31-4.

36. Casajuana J. En búsqueda de la eficiencia: dejar de hacer para poder hacer. FMC. 2005;12:579-81.

37. Reuben DB. Saving primary care. Am J Med. 2007;120:99-102.

38. Goroll AH, Berenson RA, Schoenbaum SC, Gadner LB. Fundamental reform of payment for adult primary care: Comprehensive payment for comprehensive care. $\mathrm{J}$ Gen Intern Med. 2007;22:410-5.

39. Jiwa M, Ec HC, Beilby JJ. Will promoting general practitioners with special interests threaten access to primary care? MJA. 2007;187:71-2. 